\title{
Evidence of premature immune aging in patients thymectomized during early childhood
}

\author{
Delphine Sauce, ${ }^{1}$ Martin Larsen, ${ }^{1}$ Solène Fastenackels, ${ }^{1}$ Anne Duperrier, ${ }^{2}$ Michael Keller, ${ }^{3}$ \\ Beatrix Grubeck-Loebenstein, ${ }^{3}$ Christophe Ferrand, ${ }^{2}$ Patrice Debré, ${ }^{1}$ \\ Daniel Sidi, ${ }^{4}$ and Victor Appay ${ }^{1}$

\begin{abstract}
${ }^{1}$ Infections and Immunity, INSERM UMRS 945, Avenir Group, Hôpital Pitié-Salpêtrière, UPMC University of Paris 06, Paris, France. 2INSERM U645, Etablissement Français du Sang Bourgogne/Franche-Comté, Besançon, France. ${ }^{3}$ Institute for Biomedical Aging Research, Austrian Academy of Sciences, Innsbruck, Austria. ${ }^{4}$ Service de Cardiologie Pédiatrique, Hôpital Necker Enfants Malades, Paris, France.
\end{abstract}

\begin{abstract}
While the thymus is known to be essential for the initial production of $T$ cells during early life, its contribution to immune development remains a matter of debate. In fact, during cardiac surgery in newborns, the thymus is completely resected to enable better access to the heart to correct congenital heart defects, suggesting that it may be dispensable during childhood and adulthood. Here, we show that young adults thymectomized during early childhood exhibit an altered $T$ cell compartment. Specifically, absolute $C D 4^{+}$and $\mathrm{CD8}^{+} \mathrm{T}$ cell counts were decreased, and these $T$ cell populations showed substantial loss of naive cells and accumulation of oligoclonal memory cells. A subgroup of these young patients (22 years old) exhibited a particularly altered T cell profile that is usually seen in elderly individuals (more than 75 years old). This condition was directly related to CMV infection and the induction of strong CMV-specific $T$ cell responses, which may exhaust the naive $T$ cell pool in the absence of adequate $T$ cell renewal from the thymus. Together, these marked immunological alterations are reminiscent of the immune risk phenotype, which is defined by a cluster of immune markers predictive of increased mortality in the elderly. Overall, our data highlight the importance of the thymus in maintaining the integrity of $T$ cell immunity during adult life.
\end{abstract}

\section{Introduction}

The thymus is the major production site of T cells, whose stocks are built up during fetal and early postnatal life. However, its function diminishes after the first years of life, and although thymic output is maintained into adulthood (1), the thymus mostly degenerates into fatty tissue in elderly adults (2). To date, there has been no general consensus with regard to the importance of this organ during childhood and adulthood. As a consequence, its necessity beyond the initial production of T cells might be disregarded. In fact, open heart surgery to correct congenital heart defect (CHD) usually implies complete resection of the thymus. CHD is one of the most common defects at birth. It is estimated that 1 of every 100 babies is born with a CHD (3), so approximately 40,000 newborns may be affected in the United States each year. Of the dozens of CHDs (including septal defects, defects causing obstruction in the heart or blood vessels, cyanotic defects, or even complex abnormalities), some are mild and may need little or no medical treatment even through adulthood, however some defects are life threatening, either immediately to the newborn or over time. Up to $50 \%$ of all children born with a CHD require invasive surgery to correct the defect. Over the last 20 to 30 years, open heart surgery in newborns has become increasingly safe and has been performed more often. However, surgical access to the heart and great vessels is obstructed by the thymus, which occupies a large space within newborns and is therefore removed during the intervention.

Although there has been no report of clinical indications of immunodeficiency in thymectomized CHD patients (such as higher infec-

Conflict of interest: The authors have declared that no conflict of interest exists. Citation for this article: J. Clin. Invest. 119:3070-3078 (2009). doi:10.1172/JCI39269. tion rates) $(4,5)$, young adults thymectomized during early childhood (YATECs) represent a particularly informative group in evaluation of the importance of the thymus beyond the production of the initial $\mathrm{T}$ cell stock and in studying the long-term consequences of early thymectomy in adult life. Previous short-term surveys showed that thymectomized children have reduced $\mathrm{CD}^{+} \mathrm{T}$ cell and TCR excision circle numbers compared with age-matched controls (6-10). More recently, reduction of naive $\mathrm{CD} 4^{+} \mathrm{T}$ cell numbers and production in thymectomized patients was shown to correlate with chronological age and time since thymectomy (11). In order to examine the longterm consequences of thymectomy on $\mathrm{T}$ cell population integrity, we studied quantitative and qualitative $\mathrm{T}$ cell attributes (i.e., CD4 and CD8 $\mathrm{T}$ cell counts and subset distributions, antigen-specific $\mathrm{T}$ cell functionality, TCR repertoire, proinflammatory cytokine profile) of 25 young adults (18-26 years old) who were thymectomized during cardiac surgery shortly after birth. These donors were selected for being born with a transposition of the great vessels, which results in insufficiently oxygenated blood pumped to the body and leads to cyanosis (blue discoloration of the skin) and shortness of breath. Correction of this serious $\mathrm{CHD}$ therefore required heart surgery within 2 weeks of birth. The immune characteristics of these donors were studied in comparison with age-matched, middle-aged, and elderly controls. Our data reveal that such YATECs present characteristic signs of a prematurely aged immune system. A subgroup of donors presented exacerbated alterations, which appeared to be associated with the development of an immune response against CMV.

\section{Results}

Altered T cell profile in YATECs. Access to the great vessels for switching during open heart surgery usually involves total resection of 


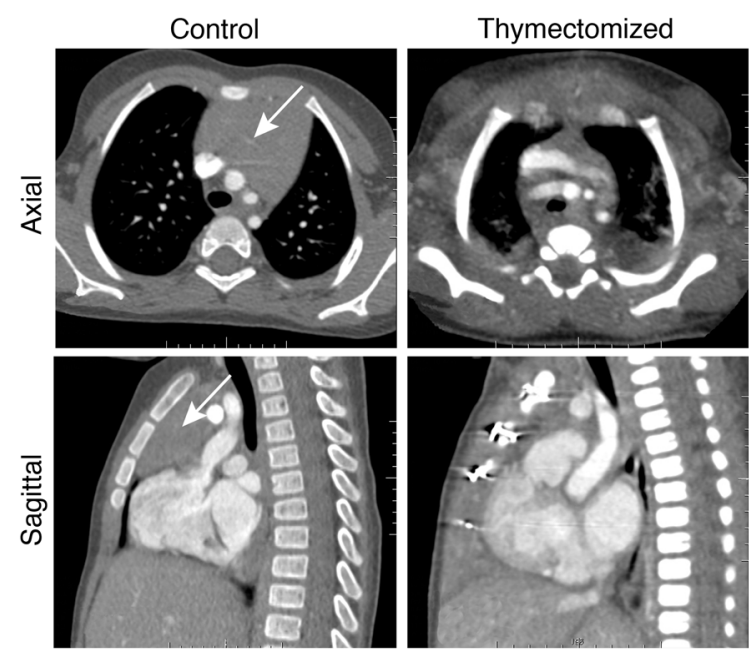

Figure 1

Durable reduction of thymic mass after early thymectomy. Representative CT scans of the heart region in a 5-year-old child thymectomized within 2 weeks of birth and a 5 -year-old control subject. Axial and sagittal sections are shown. The arrows indicate the thymus area.

the thymus. Even if residual cervical extensions and regeneration of thymic tissue in YATECs cannot be excluded, a substantial reduction of the thymic mass is evident for the years following the intervention. This is particularly obvious during childhood, when the thymus ordinarily occupies a large volume (Figure 1). Analysis of PBMC samples from YATECs, taken an average of 2 decades after thymectomy, revealed clear alterations in the $\mathrm{CD}^{+} \mathrm{T}$ cell compartment (as expected from previous studies on CHD patients; ref. $6-11$ ) as well as in the $\mathrm{CD}^{+} \mathrm{T}$ cell compartment. We found decreased $\mathrm{CD}^{+}$and $\mathrm{CD}^{+} \mathrm{T}$ cell counts in YATECs compared with age-matched controls, and even compared with middle-aged adult controls (Figure 2A). In contrast, counts of NK cells, which do not undergo thymic education, were not significantly different. In line with a lower thymic output, YATECs displayed significant reductions in the proportions of naive $\mathrm{CD}^{+}$and $\mathrm{CD}^{+} \mathrm{T}$ cells (i.e., characterized by a CD $45 \mathrm{RA}^{+} \mathrm{CCR} 7^{+} \mathrm{CD} 27^{+}$phenotype) (Figure $2 \mathrm{~B}$ ) along with lower CD31 expression (known to correlate with the TCR excision circle content in $\mathrm{CD}^{+} \mathrm{T}$ cells) $(12,13)$ within the naive $\mathrm{CD}^{+} \mathrm{T}$ cell compartment of these donors (Figure $2 \mathrm{C}$ ).

We next looked at CD57, whose expression identifies highly differentiated memory $\mathrm{CD}^{+}$and $\mathrm{CD}^{+}{ }^{+} \mathrm{T}$ cells with shorter telomere length and reduced capacity to proliferate (reviewed in ref. 14). CD57 expression is considered a marker of T cell replicative history, identifying cells that approach senescence $(15,16)$. Proportions of CD57-expressing memory $\mathrm{CD}^{+}{ }^{+}$and $\mathrm{CD}^{+} \mathrm{T}$ cells in YATECs were variable. On the whole, there was no statistical difference between YATECs and age-matched controls. Nonetheless, a number of YATECs presented levels of CD57-expressing cells equivalent to those found in the elderly group (Figure 2D). Moreover, the accumulation of CD57-expressing memory cells in YATECs correlated with decreased proportions of naive $\mathrm{CD}^{+}$and $\mathrm{CD}^{+}$ $\mathrm{T}$ cells (Figure 2E). We observed no significant difference between the proportions of regulatory $\mathrm{T}$ cells $\left(\mathrm{CD} 4^{+} \mathrm{CD} 25^{\mathrm{hi}} \mathrm{FoxP} 3^{\mathrm{hi}}\right)$ in YATECs and age-matched controls, although there was a clear trend toward a reduced absolute number in YATECs (which may be related to the lower count of $\mathrm{CD}^{+} \mathrm{T}$ cells) (data not shown).
Of note, YATECs presented higher levels of the proinflammatory cytokines IL-1 $\beta$, IL-8, and eotaxin in the plasma compared with age-matched controls (Figure 3). Higher levels of these cytokines are evidence of increased inflammation, which usually occurs with age. Overall, early thymectomy resulted in a number of immunological alterations (i.e., reduced $\mathrm{T}$ cell counts, skewed distribution of $\mathrm{T}$ cell subsets, and increased markers of inflammation) that normally occur later in life.

Normal functional attributes of virus-specific T cells. We next wanted to determine the potential impact of early thymectomy on $\mathrm{T}$ cell qualitative attributes, since they are considered crucial for efficacy in infectious or cancerous settings $(17,18)$. For this purpose, we assessed ex vivo phenotypic and functional attributes of existing virus-specific T cells in YATECs. EBV- or CMV-specific CD8 ${ }^{+}$ $\mathrm{T}$ cells were identified using a range of tetramers in YATECs in order to study their phenotype. These cells displayed regular differentiation phenotypes $(19,20)$ similar to those of controls (i.e., predominantly $\mathrm{CD} 27^{+} \mathrm{CD} 57^{-}$[less differentiated] or $\mathrm{CD} 27^{-} \mathrm{CD} 57^{+}$ [more differentiated] for EBV- and CMV-specific CD8 ${ }^{+} \mathrm{T}$ cells, respectively) (Figure 4, A and B). This suggests that thymectomy had no apparent impact on the development of memory $\mathrm{T}$ cell populations specific for persistent viruses. In line with this observation, we found no difference in terms of STAT5 phosphorylation within $\mathrm{CD}^{+}$and $\mathrm{CD} 4{ }^{+} \mathrm{T}$ cells upon stimulation with recombinant human IL-2 (rhIL-2) or rhIL-7 between YATECs and controls (data not shown), indicating that signaling to homeostatic cytokines such as IL-2 or IL-7 is not impaired following thymectomy.

Upon ex vivo stimulation with optimized epitopes, EBV-specific $\mathrm{CD}^{+} \mathrm{T}$ cells from YATECs were capable of producing antiviral factors normally (Figure 4C). Their polyfunctional profile (i.e., simultaneous degranulation and cytokine secretion) did not differ from those of controls (Figure 4D). Similarly, assessment of $\mathrm{CMV}$-specific $\mathrm{CD}^{+}$or $\mathrm{CD}^{+} \mathrm{T}$ cell polyfunction after stimulation with pp65 or IE1 overlapping peptides revealed no significant difference between YATECs and controls (Figure 4E). Last, cytokine secretion profiles upon stimulation of $\mathrm{T}$ cells with the superantigen Staphylococcal enterotoxin B were also similar between YATECs and controls (data not shown). Overall, no alteration of $\mathrm{T}$ cell functional quality was observed, suggesting that $\mathrm{T}$ cell efficacy is preserved in the setting of early thymectomy, at least during the first 20-25 years of life.

Identification of a subgroup of YATECs with marked alterations of the T cell compartment. The involution of the thymus over time is associated with a decrease in naive $\mathrm{T}$ cell production. Normal donors can be readily classified into age groups based on the concomitant reduction in naive $\mathrm{CD}^{+}$and $\mathrm{CD}^{+} \mathrm{T}$ cell proportions (Figure $5 \mathrm{~A}$ ), with individuals over 75 years old being more likely to have less than $20 \%$ of both naive $\mathrm{CD}^{+}$and $\mathrm{CD}^{+} \mathrm{T}$ cells $(P=0.001)$. On the whole, YATECs presented an altered distribution of $\mathrm{T}$ cells between naive and memory compartments, but variability among patients was relatively high, and heterogeneous $\mathrm{T}$ cell profiles were observed (Figure 2). We identified a subgroup of thymectomized donors $(n=8 ; 32 \%)$ with a particularly unbalanced T cell distribution that is usually characteristic of people over 75 years old (Figure 5A). Among all YATECs, these donors also had the lowest CD4 T cell counts $(P=0.03)$.

The TCR repertoire of sorted $\mathrm{CD}^{+}$and $\mathrm{CD} 8^{+} \mathrm{T}$ cell populations from these donors was analyzed by TCR V $\beta$ (TCRBV) spectratype analysis (Figure $5 \mathrm{~B}$ ). The analysis revealed a marked reduction in diversity within the $\mathrm{CD} 8^{+} \mathrm{T}$ cell compartment compared with age- 

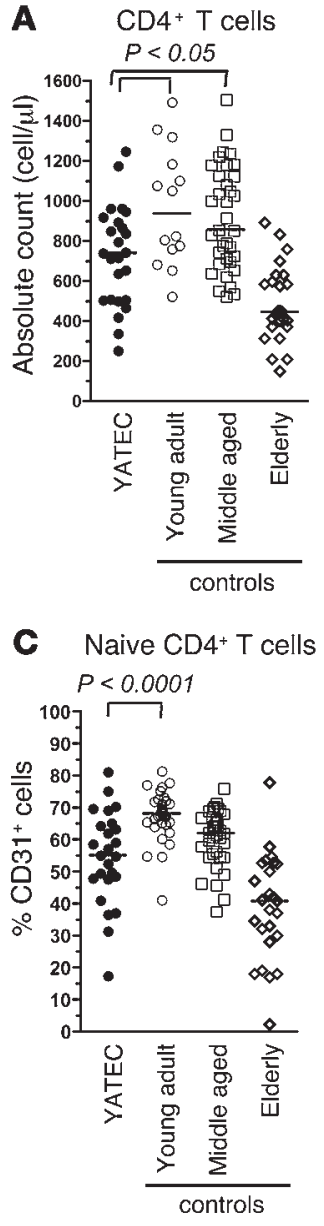
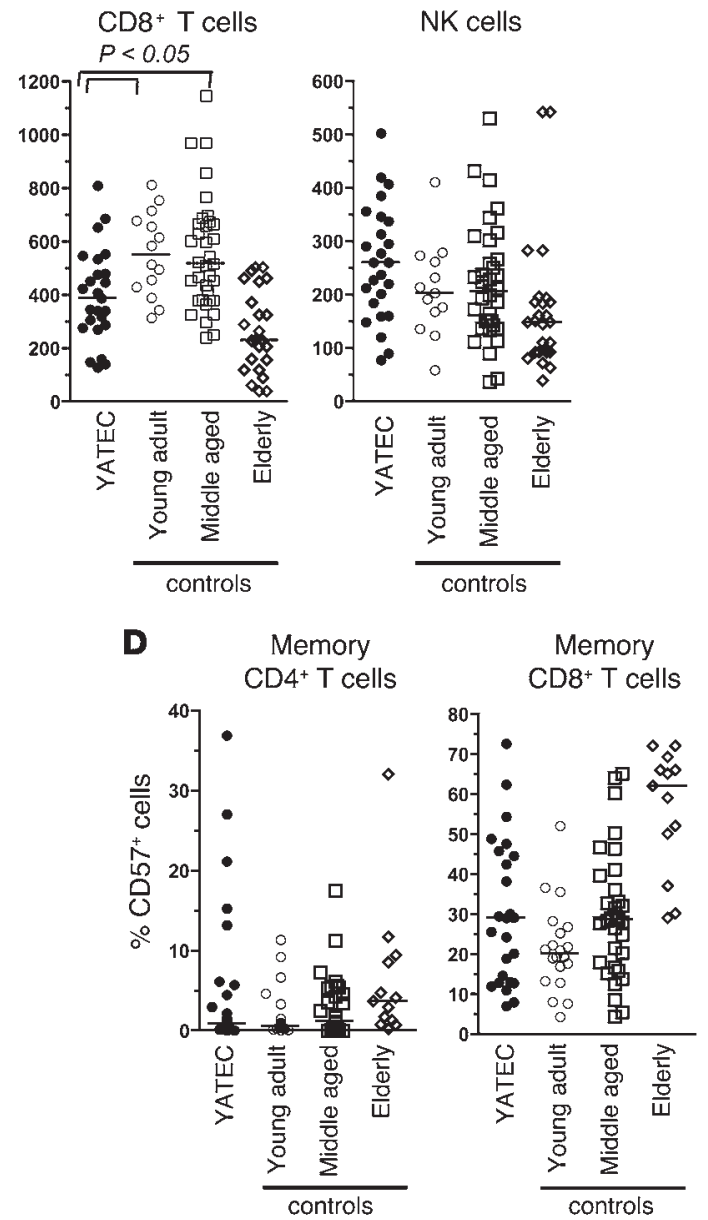

B $\quad \mathrm{CD} 4+\mathrm{T}$ cells
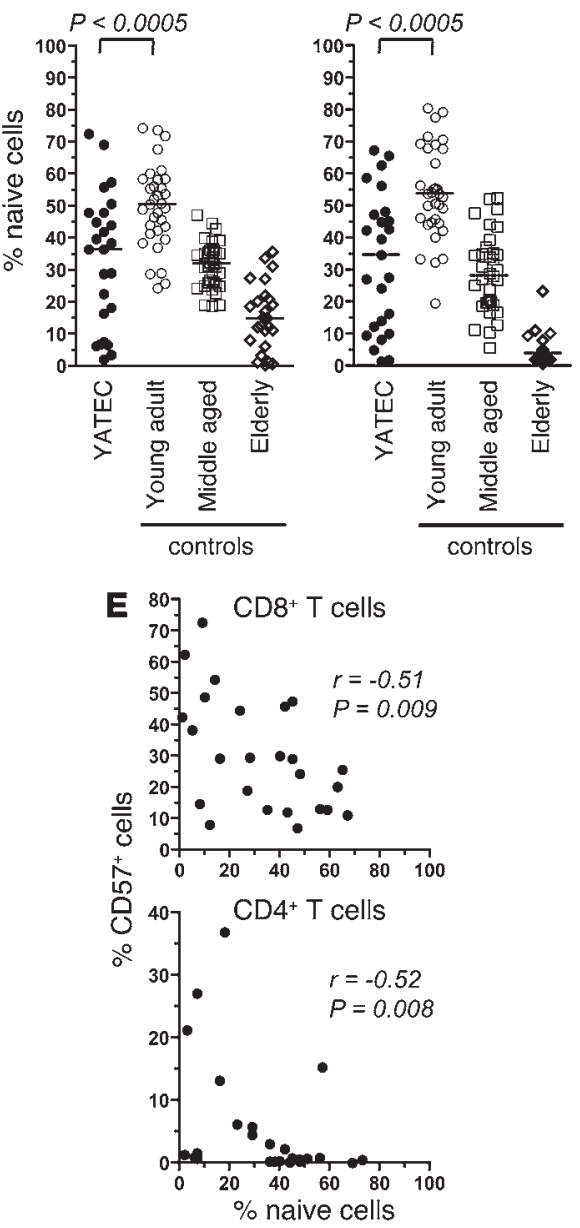

Figure 2

T cell count and subset distribution in thymectomized adults. (A) Absolute counts for CD4+, CD8+ T cells and NK cells in YATECs (median age, 22.0 years). Counts in young adult (median age, 21.9 years), middle-aged (median age, 35.3 years), and elderly (median age, 82 years) controls are shown for comparison. (B) Proportions of naive $\left(\mathrm{CD} 45 \mathrm{RA}{ }^{+} \mathrm{CCR} 7^{+} \mathrm{CD} 27^{+}\right) \mathrm{CD} 4^{+}$or $\mathrm{CD} 8^{+} \mathrm{T}$ cells in YATECs and young, middle-aged, or elderly controls. (C) Proportions of CD31+ naive CD4+ $\mathrm{T}$ cells. (D) Proportions of CD57+ cells within memory CD4+ ${ }^{+}$or CD8 ${ }^{+} \mathrm{T}$ cells. (E) Correlation between naive and $\mathrm{CD} 57^{+}$memory $\mathrm{CD} 4^{+}$or $\mathrm{CD} 8^{+} \mathrm{T}$ cell percentages in YATECs. The horizontal lines in A-D indicate the median. $P$ values were calculated by the Mann-Whitney $U$ test for group comparisons. Spearman's rank test was used to determine correlations.

matched controls or YATECs with mild T cell alteration, with a majority of $\mathrm{V} \beta$ families affected and clonality indexes drawing near those of individuals of old age (Figure 5C). Surprisingly, on the whole, only a few $\mathrm{CD}^{+} \mathrm{T}$ cell $\mathrm{V} \beta$ families were actually affected, indicating that early thymectomy or advanced age had only a modest impact on $\mathrm{CD}^{+} \mathrm{T}$ cell repertoire diversity despite the apparently low levels of naive $\mathrm{CD}^{+} \mathrm{T}$ cells. Discrepancy between $\mathrm{CD} 4^{+}$and $\mathrm{CD}^{+} \mathrm{T}$ cell oligoclonality (as well as size of the $\mathrm{CD} 57^{+}$subset; see Figure 2D), observed in both the YATEC and elderly groups, likely reflects the disparate outcome to antigenic stimulation for $\mathrm{CD}^{+}$ versus $\mathrm{CD}^{+} \mathrm{T}$ cells, as described in the literature (21-28). $\mathrm{CD}^{+}$ $\mathrm{T}$ cells are known to undergo extensive expansion upon antigenic stimulation and then rest to resupply the resting memory $\mathrm{CD}^{+}$ $\mathrm{T}$ cell pool. In contrast, antigen-driven expansion of memory $\mathrm{CD}^{+}$ $\mathrm{T}$ cells is limited, and fewer $\mathrm{CD} 4^{+} \mathrm{T}$ cells are able to survive after activation, which may be related to an intrinsically lower capacity for survival (29). Overall, this may account for the clear loss of $\mathrm{TCR}$ repertoire diversity in the $\mathrm{CD} 8^{+} \mathrm{T}$ cell population (in contrast to $\mathrm{CD}^{+} \mathrm{T}$ cells), which reflects the accumulation of oligoclonal memory $\mathrm{T}$ cell populations in the periphery.

Association with CMV infection. We next aimed to identify factors associated with this marked immune alteration in order to provide mechanistic insights into its development in some YATECs. All YATECs had comparable socioeconomic backgrounds and medical histories (including standard records of vaccination). Examination of CT scans from YATECs did not permit us to determine whether the development of such altered immune phenotypes was influenced by the extent of thymic resection during cardiac intervention and the presence of residual thymic tissue. Instead, we looked for extrinsic causative factors, which may potentially drive the expansion of oligoclonal $\mathrm{T}$ cell populations. Interestingly, accumulation of highly differentiated memory $\mathrm{CD}^{+} \mathrm{T}$ cells in normal donors has been linked to prior CMV infection (but not infection with EBV, varicella-zoster virus, or the attenuated measles-mumps-rubella vaccine strains) (30). While $48 \%$ of our study group was CMV seropositive, in keeping with the normal 


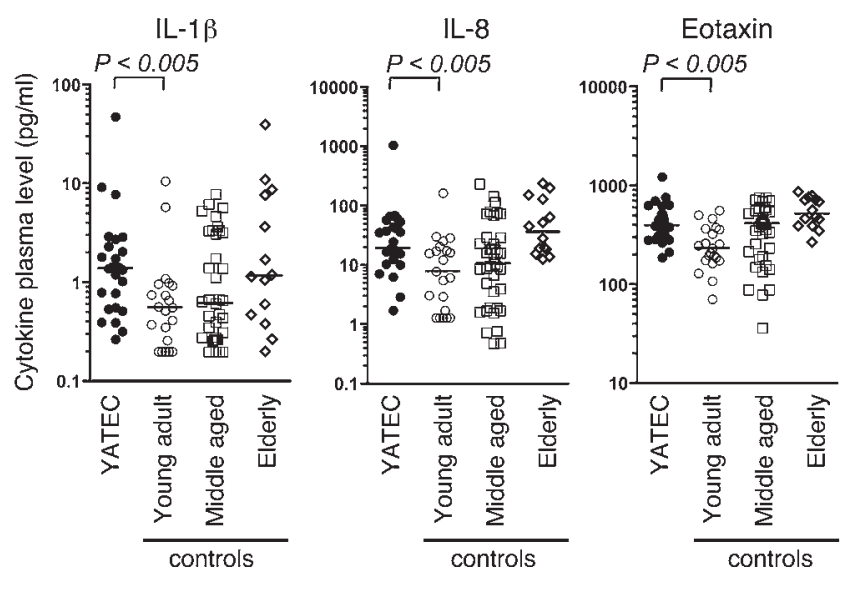

Figure 3

Proinflammatory cytokine profile in YATECs. Plasma levels of the proinflammatory cytokines IL-1 $\beta$, IL-8, and eotaxin in YATECs, and young adult, middle-aged, or elderly control subjects. The horizontal lines indicate the median. $P$ values were calculated using the MannWhitney $U$ test for group comparisons.

population of this age (51\% in our age-matched control group), we found a discrepancy between YATECs with mild versus strong alterations, with a higher occurrence of CMV seropositivity in the latter group ( $88 \%$ vs. 36\%; Figure 6A). We then measured the magnitude of IFN- $\gamma$-producing pp65- or IE1-specific T cells in all YATECs in order to get a relative picture of the total frequency of CMV-specific T cells (31). The proportions of CMV-specific T cells allowed clear discrimination between YATECs with mild versus strong alterations (Figure 6, B and C).

These results suggest that the establishment of a strong CMVspecific $\mathrm{T}$ cell response may cause marked alterations of the $\mathrm{T}$ cell compartment in the context of lack of adequate thymic output. In order to further highlight this point, we then considered the occurrence of a CMV-specific T cell response as a criteria to compare age-matched controls and YATECs. Despite similar levels of IFN- $\gamma$-producing CMV-specific (pp65 or IE1) T cells in the 2 groups (i.e., CMV-infected YATECs or controls) (Figure 6D), YATECs had significantly lower percentages of naive $\mathrm{CD}^{+}$or $\mathrm{CD}^{+} \mathrm{T}$ cells (Figure $6 \mathrm{E}$ ). Of note, thymectomy or CMV seropositivity alone appeared to have an effect on naive $T$ cell proportions, although this was less marked than with the combination thymectomy and CMV seropositivity. In addition, we found negative correlations between the levels of both pp65- or IE1-specific T cells and $\mathrm{CD}^{+}$and $\mathrm{CD}^{+}$naive $\mathrm{T}$ cell proportions in YATECs (Figure $6 \mathrm{~F}$ ). These observations are in line with TCR repertoire diversity in these groups (i.e., considering $\mathrm{CMV}$ seropositivity). In retrospect, although CMV-seropositive agematched controls showed a few oligoclonal $V \beta$ families, the overall $T$ cell repertoire diversity was not significantly affected (Figure 5C). In contrast, YATECs with significant loss of TCR repertoire diversity were indeed CMV seropositive.

\section{Discussion}

The present study shows that young adults who were thymectomized within 2 weeks of birth display a number of immunological alterations, including lower $\mathrm{CD} 4^{+}$or $\mathrm{CD}^{+} \mathrm{T}$ cell counts, reduced proportions of recent thymic emigrants and naive cells, accumulation of oligoclonal memory $\mathrm{T}$ cell populations (including highly differentiated $\mathrm{CD} 7^{+}$cells), and increased markers of inflammation. The occurrence of such changes is normal with age, but it is usually observed much later during life. Our results therefore indicate that YATECs can have premature signs of immune aging and that the phenomenon of immunosenescence is strongly related to the lack of thymic activity and inadequate production of new $\mathrm{T}$ cells, independently of age. Future studies are needed to gain insight into the increase of proinflammatory cytokines in YATECs, to understand its relationship to altered homeostasis and a disequilibrium between lymphocyte subsets in these donors. For instance a number of studies suggest a role for regulatory $\mathrm{T}$ cells in maintaining intestinal homeostasis (32). An imbalance between regulatory and effector cells may thus affect the control of inflammation in the gastrointestinal tract area, potentially leading to the rise of proinflammatory markers. It is important to note that the absence of thymus appeared to have no adverse consequence on memory $\mathrm{T}$ cell generation and the functional attributes of antigen specific $\mathrm{T}$ cells, at least within $20-25$ years following thymectomy. This may account for the lack of clinical symptoms despite apparent immunological alterations at the level of T cell production in YATECs.

Despite their young age, a number of YATECs (32\%) presented particularly marked alterations in the $\mathrm{T}$ cell compartment that are distinctive of individuals older than 75 years. These alterations (i.e., profound imbalance between naive and memory $\mathrm{T}$ cell proportions, loss of $\mathrm{T}$ cell repertoire diversity, evidence of inflammation) are reminiscent of the immune risk phenotype, which is defined by gerontologists as a cluster of immune measures that are predictive of early all-cause mortality in the elderly (33, 34). Although all YATECs enrolled in the present study (including those with severe immunological alterations) were clinically asymptomatic, such phenotype is suggestive of a certain degree of immune fragility in this subgroup of YATECs. Of note, recent data suggest that thymectomized children present delayed antibody responses to tick-borne encephalitis vaccination (35). The demise of the thymus in the elderly is considered a major causative factor of declining immune competence $(2,36)$. Moreover, it is known that continuous $\mathrm{T}$ cell renewal is crucial for the maintenance of effective immunity (37). Last, loss of TCR repertoire diversity has been shown to contribute to diminished immune responsiveness $(38,39)$. Due to their relatively young age, it is likely that YATECs have only been exposed to a limited number of pathogens, against which their initial immune resources have been sufficient, so that it may be too early to document adverse clinical outcomes. Nonetheless, the consequences of thymectomy on patients' health are likely to arise earlier than normally anticipated, in relation to a reduced capacity to mount effective adaptive immune responses to new pathogens.

The occurrence of such a marked phenotype was directly associated with the development of a strong $T$ cell response against CMV. This finding is in line with data from murine studies suggesting that, in the absence of thymic contribution, the pool of naive $\mathrm{T}$ cells wanes, eventually leaving antigen-experienced $\mathrm{T}$ cells to fill the immunological space. In these conditions, the $\mathrm{T}$ cell repertoire is thus shaped by the response (i.e., T cell activation, expansion, or death) to antigens (self or foreign) (40-42). In this context, infection by CMV is particularly relevant: although it is asymptomatic in most immunocompetent subjects, it is known to induce a massive expansion of CMV-specific T cells, reaching up to $40 \%$ of total $\mathrm{T}$ cells during chronic infection (31). This phenomenon is also known as memory inflation $(43,44)$ and was recently shown to be 
A

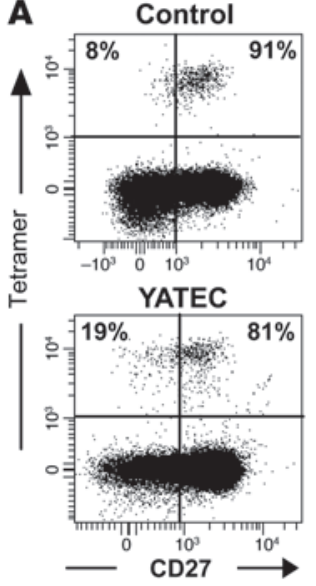

B

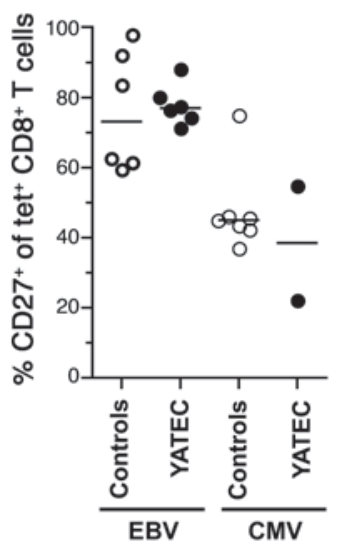

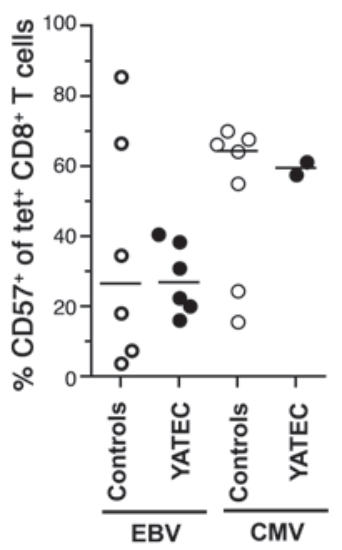

Gated on Ag-specific cells

C

Gated on $\mathrm{CD}^{+}$cells
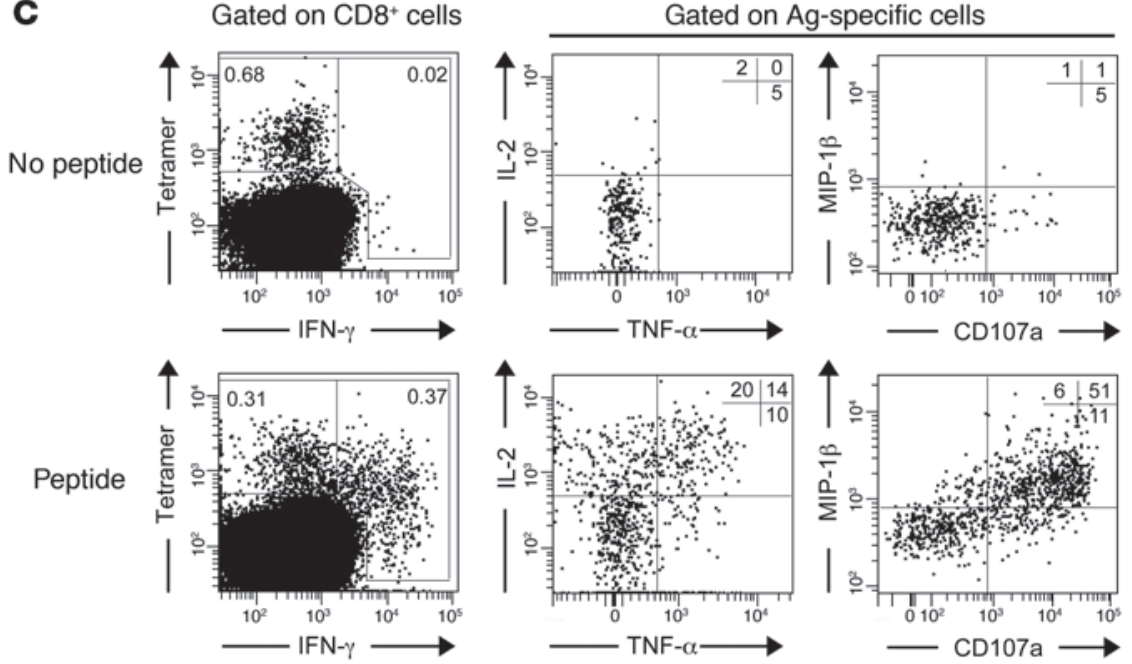

Figure 4

Phenotypic and functional assessment of virusspecific $\mathrm{CD}^{+} \mathrm{T}$ cells in YATECs. (A) Representative staining of CD27 on HLA-A11 EBV EBNA-3B AK10-specific CD8 ${ }^{+} T$ cells identified in 1 YATEC and 1 control subject. Percentages of $\mathrm{CD} 27^{-}$or $\mathrm{CD} 27^{+}$cells within tetramer-positive populations are shown. (B) Expression of the differentiation markers CD27 or CD57 on EBV- or CMV-specific CD8 ${ }^{+} \mathrm{T}$ cells, identified using tetramers in YATECs or control individuals. Horizontal bars indicate the median. (C) Representative example of simultaneous multifunctional assessment of B8-FL9 EBV-specific $C D 8^{+} \mathrm{T}$ cells from a YATEC patient. Cells were stimulated overnight in the presence of cognate peptide prior to intracellular staining and analysis using 9-color flow cytometry. Percentages of cells in the different quadrants are shown. Plots are gated on $\mathrm{CD} 3^{+} \mathrm{CD} 8^{+} \mathrm{T}$ cells and tetramer-positive T cells, respectively. (D) Polyfunctional profiling of EBV-specific CD8 ${ }^{+}$ T cell populations from YATECs $(n=8)$ or controls $(n=10)$ upon stimulation with their cognate peptide. For simplicity, percentages of cells are grouped according to the number of functions (from CD107a or CD40L, IFN- $\gamma$, TNF- $\alpha$, IL-2, and MIP-1 $\beta$ ) elicited in response to antigen encounter. Individual segments of the pie charts represent the proportions of cells within each total population that exhibited the number of functions indicated. (E) Polyfunctional profiling of CMV-specific CD8+ or CD4+ $T$ cell populations from CMV-seropositive YATECs $(n=15)$ or CMV-seropositive controls $(n=15)$ upon stimulation with pp65 and IE1 overlapping peptides.
D

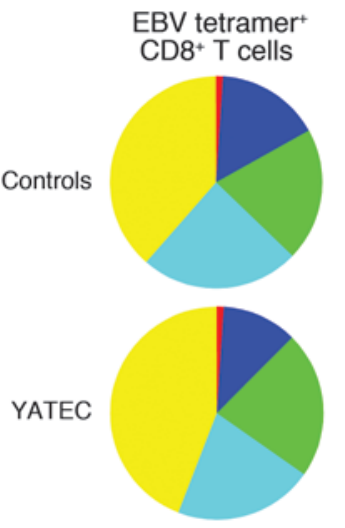

E

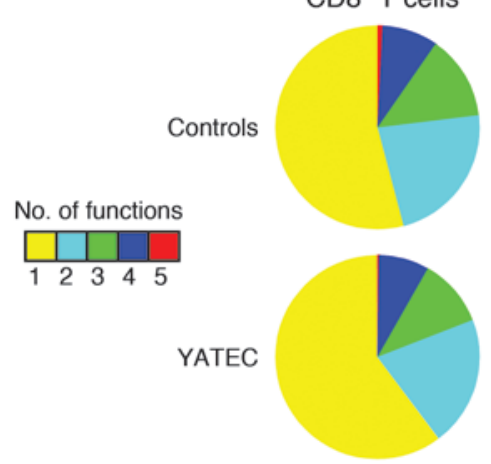

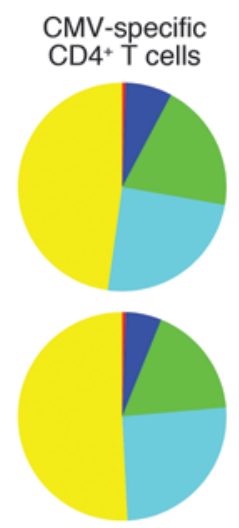

maintained by a continuous replacement of short-lived CMV-specific $T$ cells through the recruitment of naive $T$ cells in the murine CMV infection model (45). Together, these findings indicate that in order to cope with the strong pressure imposed by CMV, the cellular immune system prematurely exhausts its resources in the context of lack of adequate thymic output. Although CMV infection is usually considered benign, it has been proposed to play an important role in the development of immunosenescence (46). Our data highlight the impact of this virus on the immune sys- tem and potential consequences on immune integrity in particular contexts such as the absence of thymus. This may account at least partially for the association between CMV infection and early mortality in elderly (47) or for more rapid disease progression in HIV co-infected patients (48), settings characterized by a lack of adequate $\mathrm{T}$ cell renewal.

Importantly, since CMV prevalence is high in the general population (50\% to $80 \%$ ), a large number of CHD patients is at risk of developing significant immunological alterations. Considerate 
A

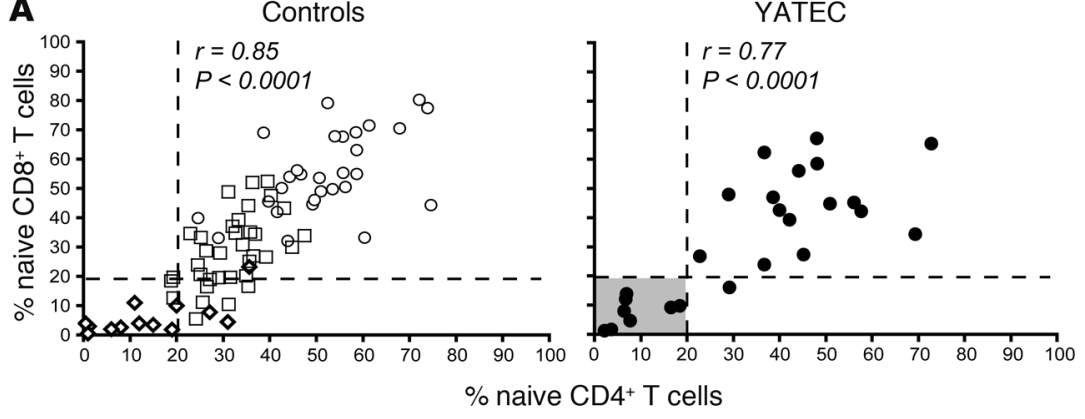

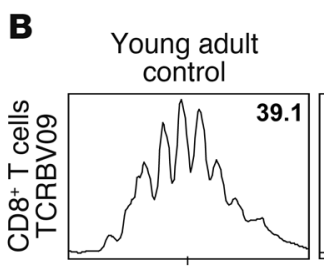

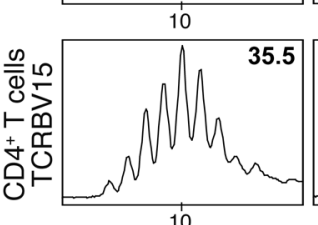

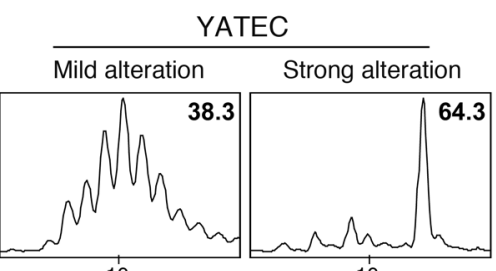

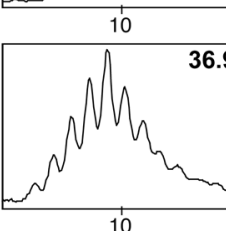

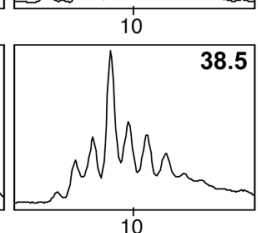
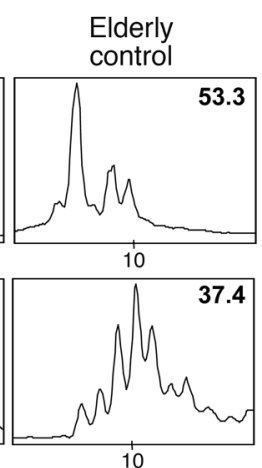

\section{Figure 5}

YATECs with marked alterations of the T cell compartment. (A) Proportions of CD4+ or CD8 ${ }^{+}$naive T cells in control age groups (open circles, young adults; open squares, middle-aged adults; and open diamonds, elderly) and YATECs (filled circles). A subgroup of YATECs with values similar to those of the elderly group is indicated by a grey square. Spearman's rank test was used to determine correlations. (B) Representative examples of $\mathrm{V} \beta$ family spectratyping analysis performed separately on CD4+ and CD8 ${ }^{+} \mathrm{T}$ cell populations from young adult controls, YATECs with mild or profound T cell alterations, and elderly controls. Numbers indicate the index of clonality for each V $\beta$ family. (C) Index of clonality for all V $\beta$ families are shown in young adult controls $(n=4)$, YATECs with mild $(n=2)$ or profound $(n=4)$ T cell alterations, and elderly controls $(n=4)$. Each symbol represents a single V $\beta$ family. + and - indicate CMV seropositivity. Horizontal bars indicate the median. $P$ values were calculated using the Mann-Whitney $U$ test.

immune and clinical check-ups of thymectomized CHD patients may therefore be advised to monitor the development of an immune risk phenotype and potentially related illnesses. Moreover, approaches to boost residual thymic activity and reconstitute the naive $\mathrm{T}$ cell compartment in thymectomized CHD patients may be considered. In this context, IL-7, a cytokine that plays an important role in modulating thymic output and the expansion of naive and memory cells may represent an interesting perspective for YATECs $(49,50)$. Indeed, recent studies show that administration of rhIL-7 increases in vivo TCR repertoire diversity through the preferential expansion of naive $\mathrm{T}$ cells in healthy individuals (51) as well as in lymphopenic HIV-infected patients $(52,53)$. Last, surgeons are highly recommended to preserve as much thymic tissue as possible when performing interventions in newborns with CHD. The maintenance of thymic activity and T cell diversity is necessary to prevent the premature development of an immune risk phenotype as these patients age and as pathogens challenge their immune system.

\section{Methods}

Study subjects and samples. Blood samples were obtained from 25 young, healthy adults (range, 18-26.2 years old; median, 22 years old) who had complete removal of the thymus within 15 days of birth during open heart
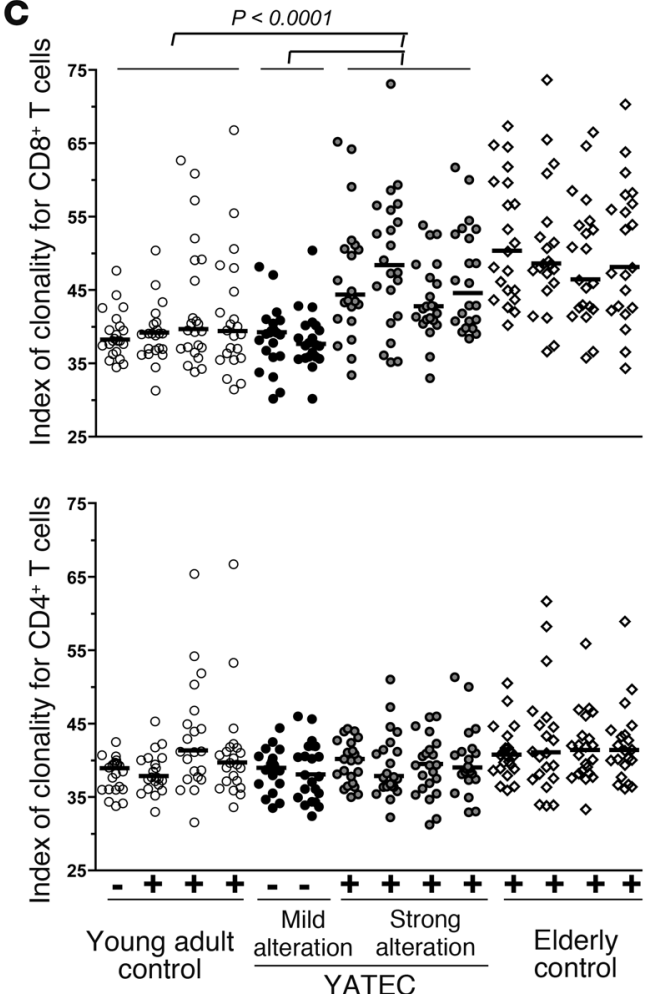

surgery due to transposition of great vessels at the Hôpital Necker Enfants Malades. Transposition of the great vessels necessitated a single operation, and none of the patients enrolled in the study were re-operated. There were no records of blood transfusions or additional early childhood illness following surgery (e.g., cyanosis). There was no genetic association with transposition of the great vessels. Thymectomy was performed by total resection of both lobes for ease of surgical access to the heart and major vessels. Recent thoracic X-ray did not reveal signs of remaining thymic tissue. Rates of hospitalization or infection did not differ from the general population of the same age. Donors were only included if they had no transplantation, hematologic disorders, immunosuppressive or cortisone therapy, or other medications known to influence the bone marrow or the immune system. The study was approved by the local institutional ethics committee (i.e., Comite de Protection des Personnes, Hôpital Pitié-Salpêtrière). All participants gave their written informed consent. For comparison, blood samples were obtained from 29 normal age-matched donors (range, 18.6-25.7 years old; median, 21.9 years old) and 35 middle-aged (range, 26.4-55 years old; median, 35.3 years old) or 26 elderly (range, 75-93; median, 82 years old) adults. Elderly individuals with malignancies, acute diseases, or advanced stages of severe chronic diseases, such as chronic inflammatory disease, atherosclerotic disease, congestive heart failure, poorly controlled diabetes mellitus, renal, or hepatic disease, or chronic obstructive pulmonary disease, as well as individuals undergoing immunosuppressive therapy were excluded from the 
A

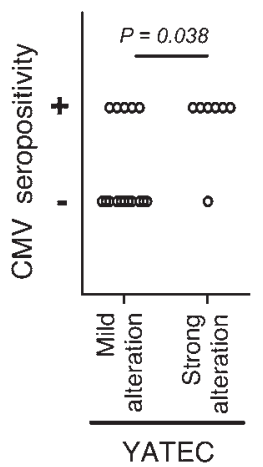

D

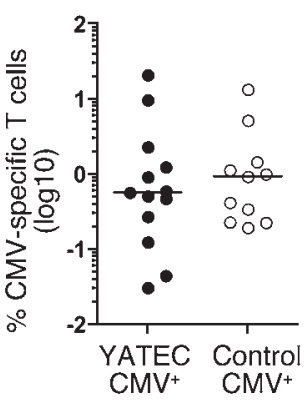

B Seronegative

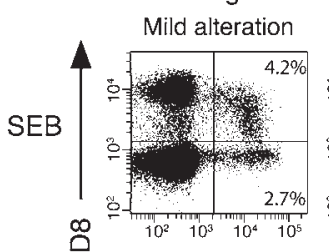

pp65

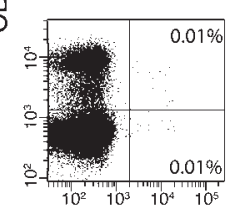

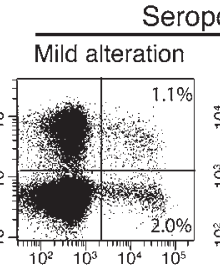

Seropositive

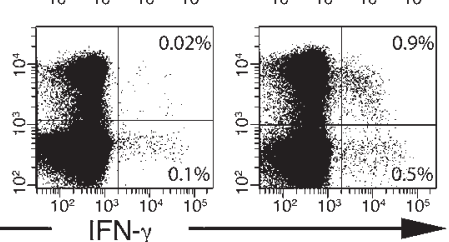

c

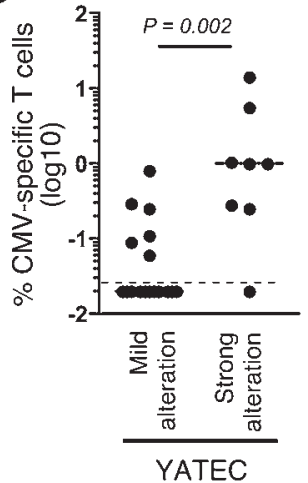

E

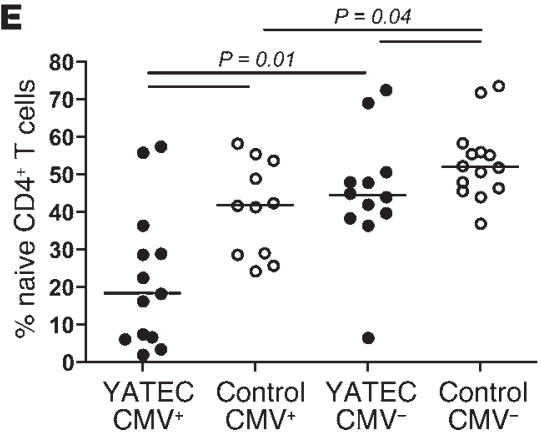

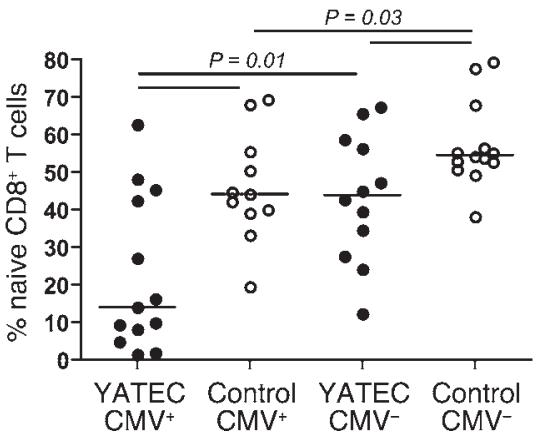
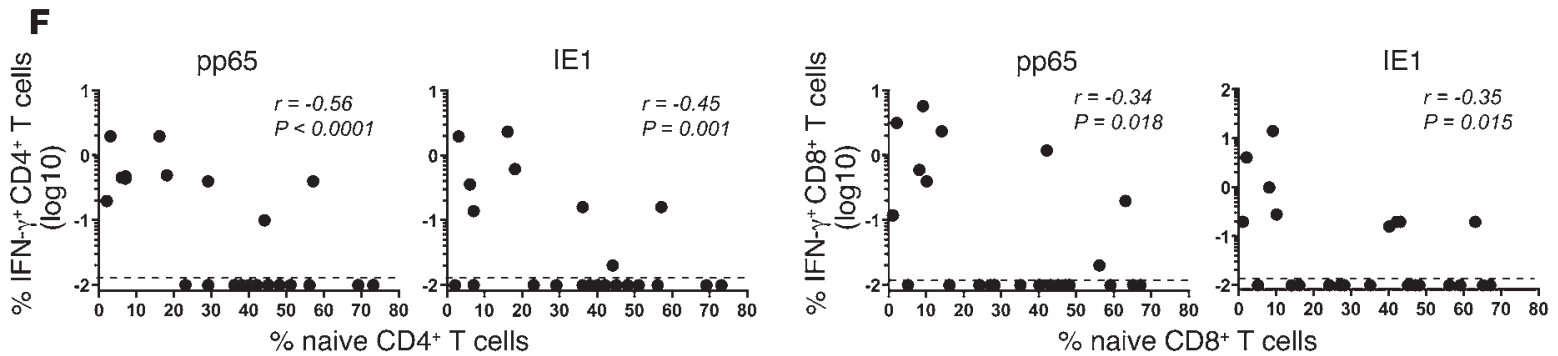

Figure 6

Association between CMV-specific T cell responses and marked T cell alterations in YATECs. (A) CMV seropositivity among YATECs with mild $(n=17)$ or strong $(n=8)$ T cell alterations. Each circle represents one patient. The $P$ value was calculated using the $\chi^{2}$ test. (B) Representative stainings for IFN- $\gamma$ in $\mathrm{CD}^{+}$or $\mathrm{CD}^{+}{ }^{+} \mathrm{T}$ cells from a CMV-seronegative YATECs or CMV-seropositive YATECs with mild or strong T cell alterations upon stimulation with Staphylococcal enterotoxin B (SEB) or pp65 overlapping peptides. Percentages of IFN- $\gamma^{+}$cells within CD8- or CD8+ populations are shown. (C) Percentages of CMV-specific (pp65 and IE1) T cells in YATECs with mild or profound T cell alterations. (D) Percentages of CMV-specific (pp65 and IE1) T cells in CMV-seropositive YATECs or control subjects. (E) Proportions of naive CD4+ or CD8 ${ }^{+} \mathrm{T}^{+}$cells in YATECs or age-matched control subjects who were CMV seropositive or seronegative. (F) Correlations between percentages of pp65- or IE1-specific $\mathrm{CD}^{+}$or $\mathrm{CD} 8^{+} \mathrm{T}$ cells and proportions of naive $\mathrm{CD} 4^{+}$or $\mathrm{CD} 8^{+} \mathrm{T}$ cells in YATECs. In $\mathrm{C}-\mathrm{E}$, horizontal bars indicate the median. In C and $\mathbf{F}$, dashed lines indicate the limit of detection. $P$ values were calculated using the Mann-Whitney $U$ test for group comparisons. Spearman's rank test was used to determine correlations.

study. Donors were HLA typed and their PBMCs purified. CMV serology was performed on plasma samples using a Mastazyme-CMV serology kit (Mast Diagnostics) according to the manufacturer's recommendations.

Reagents. Directly conjugated and unconjugated antibodies were obtained from BD Biosciences (CD4 [APC-cyanin 7], HLA-DR [PE-cyanin 7], CD25 [PE], CD57 [FITC], CCR7 [PE-cyanin 7], CD38 [APC], CD107a [cyanin 5-PE], CD40L [cyanin 5-PE], IFN- $\gamma$ [Alexa Fluor 700], and TNF- $\alpha$ [PE-cyanin 7]); Beckman Coulter (CD45RA [ECD]); Caltag (CD8 [Alexa Fluor 405]); eBioscience (Foxp3 [APC]); Dako (CD3 [cascade yellow]); and BioLegend (CD27 [Alexa Fluor 700], CD31 [Alexa Fluor 647]). Tetramers were produced as previously described (54) and included the following epi- topes: HLA-A2 CMV pp65-NV9 and IE1-VL9; HLA-A2 EBV BMLF1-GL9 and BMRF1-YV9; HLA-A11 EBV EBNA-3B AK10; HLA-B7 CMV Pp65TM10 and Pp65-RL11; HLA-B8 CMV IE1-EM9, IE1-EM10, IE1-QV9; HLA-B8 EBV BZLF1-RL8, EBNA-3A-FL9 and EBNA-3A-QL9; HLA-B35 CMV pp65-IY11; and HLA-B35 EBV EBNA-1-YM9, EBNA-3A-HY11 and BZLF1-EY11. Measures of the cytokines IL-1 $\beta$, IL-8, and eotaxin in the plasma were performed using multiplex bead immunoassays (Biosource) and a Luminex instrument. CMV-overlapping peptides were provided by Daniel Olive (Centre Paoli Calmettes, Marseille, France).

Flow cytometry and polyfunctional assessment. Cell surface marker stainings were performed as previously described (55). Briefly, titrated tetramers 
(tricolor or PE conjugated) were added to $10^{6}$ PBMCs, followed by the addition of a panel of titrated antibodies and incubated for 15 minutes at room temperature. To assess functional capacity of EBV-specific $\mathrm{CD}^{+}$ $\mathrm{T}$ cells, $10^{6}$ tetramer-prestained PBMCs were incubated in the presence of specific peptide $(5 \mu \mathrm{M})$ and anti-CD107a antibodies $(10 \mu \mathrm{l})$ for 1 hour at $37^{\circ} \mathrm{C}$ in a $5 \% \mathrm{CO}_{2}$ incubator, followed by an additional 15 hours in the presence of the secretion inhibitors monensin $(2.5 \mu \mathrm{g} / \mathrm{ml}$; Sigma-Aldrich) and Brefeldin A ( $5 \mu \mathrm{g} / \mathrm{ml}$; Sigma-Aldrich). Negative controls were obtained in the absence of peptide and prestaining with tetramers. BD Cytofix/ Cytoperm was used for permeabilization of the cells prior to staining for intracellular cytokines. The percentage frequencies of multifunctional cells were calculated within the total population of detectable antigen-specific $\mathrm{CD}^{+} \mathrm{T}$ cells. To assess functional capacity of $\mathrm{CMV}$-specific $\mathrm{CD}^{+}$or $\mathrm{CD}^{+} \mathrm{T}$ cells, PBMCs were stimulated with 15 -amino-acid-long synthetic peptides overlapping by 10 amino acids and spanning the CMV proteins pp65 and IE1. Assessment of CD107a was replaced by CD40L in the case of $\mathrm{CD} 4^{+} \mathrm{T}$ cells. The limit of detection for cytokine secretion was $0.01 \%$ in $\mathrm{CD}^{+}$or $\mathrm{CD}^{+} \mathrm{T}$ cell populations. Stainings were analyzed on a LSR2 flow cytometer (Becton Dickinson) with appropriate isotype controls and color compensation. Data were analyzed using FlowJo v8.2 (Tree Star Inc.) and DIVA software. Multifunctional data were analyzed with Pestle and Spice (provided by Mario Roederer, Vaccine Research Center, National Institute of Allergy and Infectious Diseases, NIH).

TCRBV CDR3 spectratyping. Immunomagnetic sorting of $\mathrm{CD}^{+}$and $\mathrm{CD} 4^{+}$ T cells from PBMCs was performed using MACS technology according to the manufacturer's recommendations (Miltenyi Biotech). The cells were added to RLT buffer (RNase Easy Blood Mini kit; Qiagen) with $1 \% \beta$-mercaptoethanol for CDR3 spectratyping. cDNA was amplified in 24 separate PCR reactions (V $\beta$ families 1-24), each containing 1 specific human V $\beta$ subfamily primer coupled with a $\mathrm{C} \beta$ primer recognizing the constant $\mathrm{BC} 1$ and $\mathrm{BC} 2$ of the $\beta$ chain, as previously described (56). Data were analyzed with Immunoscope 3.1a and ISEApeaks software. The nomenclature of TCRBV segments proposed by Arden et al. (57) was used in this study. For each subject, we then calculated the index of clonality of each $V \beta$ family as the square root of the sum of squares of the normalized area at each peak. Thus, indexes of clonality were established for each $V \beta$ family, for each donor, and separately for $\mathrm{CD}^{+}$and $\mathrm{CD}^{+} \mathrm{T}$ cells.

Statistics. Statistical analysis was performed using GraphPad prism software. Data were compared using the Mann-Whitney $U$ test. Spearman's rank test was used to determine correlations. $P$ values at or below 0.05 were considered significant.

\section{Acknowledgments}

We are very grateful to the patients and staff of the pediatric cardiology department at the Necker Hospital in Paris, in particular Pascal Vouhe and Phalla Ou. We are indebted to the T4/T8 laboratory (Hospital Pitié-Salpêtrière), headed by Brigitte Autran and Guislaine Carcelain, for performing cell counts. We thank Martina Prelog (Innsbruck Medical University, Innsbruck, Austria) for constructive comments on the manuscript. This work was supported by an INSERM AVENIR grant, the French ANRS, Sidaction, and the European program AutoCell (LSHP-CT-2005-018953).

Received for publication March 20, 2009, and accepted in revised form July 16, 2009.

Address correspondence to: Victor Appay, Cellular Immunology Laboratory, INSERM U945, Hôpital Pitié-Salpêtrière, Paris, France. Phone: 33-1-40-77-81-83; Fax: 33-1-42-17-74-90; E-mail: victor.appay@upmc.fr.
1. Douek, D.C., et al. 1998. Changes in thymic function with age and during the treatment of HIV infection. Nature. 396:690-695.

2. Gruver, A.L., Hudson, L.L., and Sempowski, G.D. 2007. Immunosenescence of ageing. J. Pathol. 211:144-156.

3. Hoffman, J.I., and Kaplan, S. 2002. The incidence of congenital heart disease. J. Am. Coll. Cardiol. 39:1890-1900.

4. Rubinstein, A., Pelet, B., and Schweizer, V. 1976. Immunological decay in thymectomized infants. Helv. Paediatr. Acta. 30:425-433.

5. Moretta, L., et al. 1977. Imbalances in T cell subpopulations associated with immunodeficiency and autoimmune syndromes. Eur. J. Immunol. 7:696-700.

6. Wells, W.J., Parkman, R., Smogorzewska, E., and Barr, M. 1998. Neonatal thymectomy: does it affect immune function? J. Thorac. Cardiovasc. Surg. 115:1041-1046.

7. Eysteinsdottir, J.H., et al. 2004. The influence of partial or total thymectomy during open heart surgery in infants on the immune function later in life. Clin. Exp. Immunol. 136:349-355.

8. Madhok, A.B., et al. 2005. Levels of recent thymic emigrant cells decrease in children undergoing partial thymectomy during cardiac surgery. Clin. Diagn. Lab. Immunol. 12:563-565.

9. Torfadottir, H., et al. 2006. Evidence for extrathymic $T$ cell maturation after thymectomy in infancy. Clin. Exp. Immunol. 145:407-412.

10. Ogle, B.M., et al. 2006. Effacing of the T cell compartment by cardiac transplantation in infancy. J. Immunol. 176:1962-1967.

11. Prelog, M., et al. 2009. Thymectomy in early childhood: Significant alterations of the CD4(+)CD45RA(+)CD62L(+) T cell compartment in later life. Clin. Immunol. 130:123-132.
12. Kimmig, S., et al. 2002. Two subsets of naive T helper cells with distinct $T$ cell receptor excision circle content in human adult peripheral blood. J. Exp. Med. 195:789-794.

13. Junge, S., et al. 2007. Correlation between recent thymic emigrants and CD31+ (PECAM-1) CD4+ T cells in normal individuals during aging and in lymphopenic children. Eur. J. Immunol. 37:3270-3280.

14. Appay, V., van Lier, R.A., Sallusto, F., and Roederer, M. 2008. Phenotype and function of human T lymphocyte subsets: consensus and issues. Cytometry $A$. 73:975-983.

15. Brenchley, J.M., et al. 2003. Expression of CD57 defines replicative senescence and antigeninduced apoptotic death of CD8+ T cells. Blood. 101:2711-2720.

16. Papagno, L., et al. 2004. Immune Activation and CD8(+) T-Cell Differentiation towards Senescence in HIV-1 Infection. PLoS Biol. 2:E20.

17. Appay, V., Douek, D.C., and Price, D.A. 2008. CD8+ $\mathrm{T}$ cell efficacy in vaccination and disease. Nat. Med. 14:623-628.

18. Seder, R.A., Darrah, P.A., and Roederer, M. 2008. T-cell quality in memory and protection: implications for vaccine design. Nat. Rev. Immunol. 8:247-258.

19. Appay, V., et al. 2002. Memory CD8+ T cells vary in differentiation phenotype in different persistent virus infections. Nat. Med. 8:379-385.

20. van Lier, R.A., ten Berge, I.J., and Gamadia, L.E. 2003. Human CD8(+) T-cell differentiation in response to viruses. Nat. Rev. Immunol. 3:931-939.

21. Opferman, J.T., Ober, B.T., and Ashton-Rickardt, P.G. 1999. Linear differentiation of cytotoxic effectors into memory $\mathrm{T}$ lymphocytes. Science. 283:1745-1748.

22. Hu, H., et al. 2001. CD4(+) T cell effectors can become memory cells with high efficiency and without further division. Nat. Immunol. 2:705-710.
23. Homann, D., Teyton, L., and Oldstone, M.B. 2001. Differential regulation of antiviral T-cell immunity results in stable CD8+ but declining CD4+ T-cell memory. Nat. Med. 7:913-919.

24. Foulds, K.E., et al. 2002. Cutting edge: CD4 and CD8 $\mathrm{T}$ cells are intrinsically different in their proliferative responses. J. Immunol. 168:1528-1532.

25. Hayashi, N., Liu, D., Min, B., Ben-Sasson, S.Z., and Paul, W.E. 2002. Antigen challenge leads to in vivo activation and elimination of highly polarized TH1 memory T cells. Proc. Natl. Acad. Sci. U. S. A. 99:6187-6191.

26. Badovinac, V.P., Porter, B.B., and Harty, J.T. 2002. Programmed contraction of CD8(+) T cells after infection. Nat. Immunol. 3:619-626.

27. Wu, C.Y., et al. 2002. Distinct lineages of $\mathrm{T}(\mathrm{H}) 1$ cells have differential capacities for memory cell generation in vivo. Nat. Immunol. 3:852-858.

28. Tham, E.L., and Mescher, M.F. 2002. The poststimulation program of CD4 versus CD8 T cells (death versus activation-induced nonresponsiveness). J. Immunol. 169:1822-1828.

29. Ferreira, C., et al. 2000. Differential survival of naive CD4 and CD8 T cells. J. Immunol. 165:3689-3694.

30. Kuijpers, T.W., et al. 2003. Frequencies of circulating cytolytic, CD45RA+CD27-, CD8+ T lymphocytes depend on infection with CMV. J. Immunol. 170:4342-4348.

31. Sylwester, A.W., et al. 2005. Broadly targeted human cytomegalovirus-specific CD4+ and CD8+ T cells dominate the memory compartments of exposed subjects. J. Exp. Med. 202:673-685.

32. Izcue, A., Coombes, J.L., and Powrie, F. 2006. Regulatory T cells suppress systemic and mucosal immune activation to control intestinal inflammation. Immunol. Rev. 212:256-271.

33. Ferguson, F.G., Wikby, A., Maxson, P., Olsson, J., and Johansson, B. 1995. Immune parameters in 
a longitudinal study of a very old population of Swedish people: a comparison between survivors and nonsurvivors. J. Gerontol. A Biol. Sci. Med. Sci. 50:B378-B382.

34. Wikby, A., Maxson, P., Olsson, J., Johansson, B., and Ferguson, F.G. 1998. Changes in CD8 and CD4 lymphocyte subsets, $\mathrm{T}$ cell proliferation responses and non-survival in the very old: the Swedish longitudinal OCTO-immune study. Mech. Ageing Dev. 102:187-198.

35. Prelog, M., et al. 2008. Diminished response to tickborne encephalitis vaccination in thymectomized children. Vaccine. 26:595-600.

36. Naylor, K., et al. 2005. The influence of age on $\mathrm{T}$ cell generation and TCR diversity. J. Immunol. 174:7446-7452.

37. Vezys, V., et al. 2006. Continuous recruitment of naive $T$ cells contributes to heterogeneity of antiviral CD8 T cells during persistent infection. J. Exp. Med. 203:2263-2269.

38. Yager, E.J., et al. 2008. Age-associated decline in $\mathrm{T}$ cell repertoire diversity leads to holes in the repertoire and impaired immunity to influenza virus. J. Exp. Med. 205:711-723.

39. Nikolich-Zugich, J., Slifka, M.K., and Messaoudi, I. 2004. The many important facets of T-cell repertoire diversity. Nat. Rev. Immunol. 4:123-132.

40. La Gruta, N.L., Driel, I.R., and Gleeson, P.A. 2000. Peripheral $\mathrm{T}$ cell expansion in lymphopenic mice results in a restricted $\mathrm{T}$ cell repertoire. Eur. J. Immunol. 30:3380-3386.

41. Miller, N.E., Bonczyk, J.R., Nakayama, Y., and Suresh, M. 2005. Role of thymic output in regu- lating CD8 T-cell homeostasis during acute and chronic viral infection. J. Virol. 79:9419-9429.

42. Bourgeois, C., Hao, Z., Rajewsky, K., Potocnik, A.J., and Stockinger, B. 2008. Ablation of thymic export causes accelerated decay of naive CD4 T cells in the periphery because of activation by environmental antigen. Proc. Natl. Acad. Sci. U. S. A. 105:8691-8696.

43. Karrer, U., et al. 2003. Memory inflation: continuous accumulation of antiviral CD8 $+\mathrm{T}$ cells over time. J. Immunol. 170:2022-2029.

44. Holtappels, R., Pahl-Seibert, M.F., Thomas, D., and Reddehase, M.J. 2000. Enrichment of immediateearly 1 (m123/pp89) peptide-specific CD8 T cells in a pulmonary CD62L(lo) memory-effector cell pool during latent murine cytomegalovirus infection of the lungs. J. Virol. 74:11495-11503.

45. Snyder, C.M., et al. 2008. Memory inflation during chronic viral infection is maintained by continuous production of short-lived, functional $\mathrm{T}$ cells. Immunity. 29:650-659.

46. Pawelec, G., Koch, S., Franceschi, C., and Wikby, A. 2006. Human immunosenescence: does it have an infectious component? Ann. N. Y. Acad. Sci. 1067:56-65.

47. Koch, S., Solana, R., Dela Rosa, O., and Pawelec, G. 2006. Human cytomegalovirus infection and T cell immunosenescence: a mini review. Mech. Ageing Dev. 127:538-543.

48. Webster, A., et al. 1992. Cytomegalovirus (CMV) infection, CD4+ lymphocyte counts and the development of AIDS in HIV-1-infected haemophiliac patients. Clin. Exp. Immunol. 88:6-9.

49. Schluns, K.S., Kieper, W.C., Jameson, S.C., and
Lefrancois, L. 2000. Interleukin-7 mediates the homeostasis of naive and memory CD8 T cells in vivo. Nat. Immunol. 1:426-432.

50. Tan, J.T., et al. 2001. IL-7 is critical for homeostatic proliferation and survival of naive T cells. Proc. Natl. Acad. Sci. U. S. A. 98:8732-8737.

51. Sportes, C., et al. 2008. Administration of rhIL-7 in humans increases in vivo TCR repertoire diversity by preferential expansion of naive $T$ cell subsets. J. Exp. Med. 205:1701-1714.

52. Levy, Y., et al. 2009. Enhanced T cell recovery in HIV-1-infected adults through IL-7 treatment. J. Clin. Invest. 119:997-1007.

53. Sereti, I., et al. 2009. IL-7 administration drives T cell cycle entry and expansion in HIV-1 infection. Blood. 113:6304-6314.

54. Altman, J.D., et al. 1996. Phenotypic analysis of antigen-specific T lymphocytes [erratum 1998, 280:1821]. Science. 274:94-96.

55. Appay, V., and Rowland-Jones, S.L. 2002. The assessment of antigen-specific CD8 $+\mathrm{T}$ cells through the combination of MHC class I tetramer and intracellular staining. J. Immunol. Methods. 268:9-19.

56. Coito, S., et al. 2004. Retrovirus-mediated gene transfer in human primary $\mathrm{T}$ lymphocytes induces an activation- and transduction/selection-dependent TCR-B variable chain repertoire skewing of gene-modified cells. Stem Cells Dev. 13:71-81.

57. Arden, B., Clark, S.P., Kabelitz, D., and Mak, T.W. 1995. Human T-cell receptor variable gene segment families. Immunogenetics. 42:455-500. 